\title{
Carotid artery stenting versus endarterectomy -no difference in long-term outcomes
}

$\mathrm{C}$ arotid artery stenosis results from a build-up of atherosclerotic plaques within the carotid artery wall and is responsible for approximately $10 \%$ of all strokes. Carotid endarterectomy, the surgical removal of fatty deposits from the vessel, has been the treatment of choice for patients with symptomatic carotid stenosis. However, emerging evidence has indicated that carotid artery stenting might be an effective and lessinvasive alternative to endarterectomy. In a multicentre, randomized controlled trial published in The Lancet, Martin Brown and colleagues sought to "definitively compare the two treatments and establish the short-term risks of stenting and the long-term outcome of patients treated with stenting in comparison to surgery."

The International Carotid Stenting Study (ICSS) is the largest trial comparing the efficacy of carotid artery stenting and carotid endarterectomy in preventing long-term stroke. The ICSS team enrolled 1,713 patients with symptomatic atherosclerotic carotid stenosis from 50 centres worldwide. All participants were over the age of 40 years and had $\geq 50 \%$ reduction in carotid artery lumen diameter. Patients were randomly assigned in a 1:1 ratio to receive either open treatment with stenting or endarterectomy. The primary end point was fatal or disabling stroke.

\section{1 ...treatment choice should be based on procedure-related risks and individual patient characteristics... 77}

After a median follow-up of 4.2 years, 52 patients in the stenting group and 49 patients in the endarterectomy group reached the primary end point; this was not signficantly different. In addition, the cumulative 5-year risk was similar between the stented and endarterectomy groups (6.4\% versus $6.5 \%$ respectively; HR 1.06 , $95 \%$ CI $0.72-1.57, P=0.77)$. However, the secondary outcome of any stroke occurred more frequently in the stented group compared with the endarterectomy group (cumulative incidence 5.1\% versus 9.5\%; HR 1.71, 95\% CI 1.28-2.30, $P=0.0003$ ). "This finding of an excess of non-disabling stroke is consistent with all the smaller trials for symptomatic carotid stenosis," explains Brown. "The new finding from our long-term study is that this difference does not translate into long-term differences in outcome." Indeed, functional outcome measured at the end of the follow-up period was not significantly different between the treatment groups. Furthermore, the stenting and surgical groups had a similar 5-year cumulative risk of ipsilateral stroke (4.7\% versus $3.4 \%$; HR 1.29, 95\% CI 0.74-2.24) and severe carotid restenosis ( $10.8 \%$ versus $8.6 \%$, HR 1.25, 95\% CI 0.89-1.75).

While the long-term outcomes of carotid artery stenting from this primary analysis seem promising, less certainty surrounds the outcomes during the initial 30-day period after the procedure. Ross Naylor, a member of the ICSS data monitoring committee, draws attention to previous randomized studies, which show that "stenting is associated with a doubling of the 30-day risk of death/any stroke, while surgery may be associated with an increased risk of perioperative myocardial infarction." Furthermore, he emphasizes that, "once the 30-day period after treatment has elapsed, both interventions have similar outcomes regarding late stroke risk." This observation raises an important question of whether stenting or surgery is the safest intervention in the early period after the onset of symptoms.

Similar uncertainties are also highlighted in an accompanying comment article by Marco Roffi, who reiterates that "the excess of periprocedural strokes has

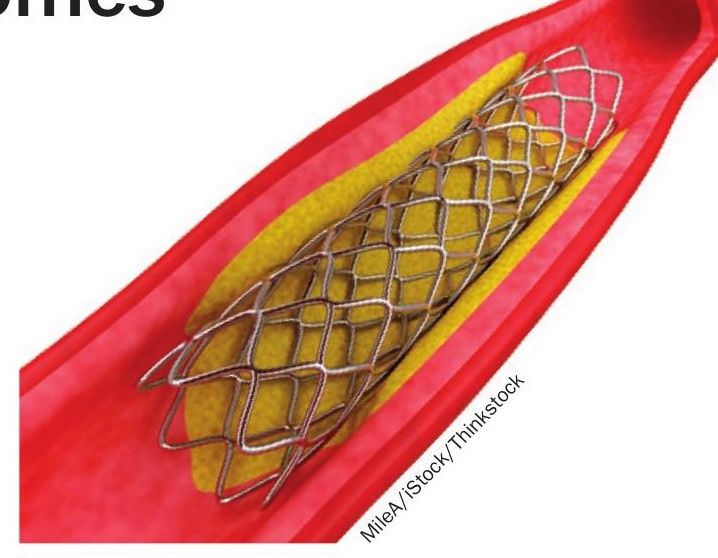

limited the acceptance of stenting as an alternative to endarterectomy." Roffi also discusses the controversy surrounding the role of operator experience in determining outcomes. The ICSS investigators argue against the claim that inexperienced proceduralists might have skewed outcomes, as the study demonstrated no differences between the relative hazards of the two procedures performed at supervised and experienced centres.

The investigators conclude that while "stenting has the disadvantage of causing more minor non-disabling strokes in the procedural period ... [this] must be weighed against the increased risk of procedural myocardial infarction, cranial nerve palsy, and access-site haematoma associated with endarterectomy." Treatment choice should be based on both procedure-related risks and individual patient characteristics. Brown suggests that "future research needs to concentrate on ways of making stenting safer, [including] better design of stents and better protection devices."

Karina Huynh 